\title{
توجيهات في فهم علم اللغة
}

\author{
مشفيك هندري \\ جامعة السلطان الشريف قاسم الإسلامية الحكومية رياو، اندونيسيا \\ muspikahendri@gmail.com
}

\section{ملخحصص}

علم اللغة. هو دراسة اللغة على نحو علمي. وتدرس اللغة في إطار علم اللغة في البجالات الآتية : الأصوات,

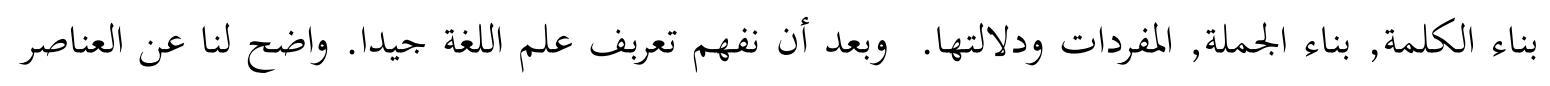

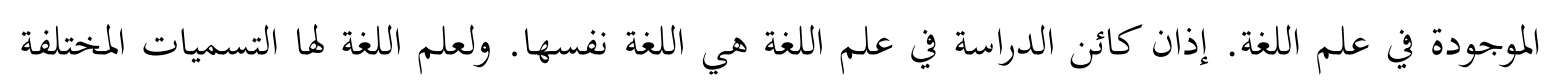

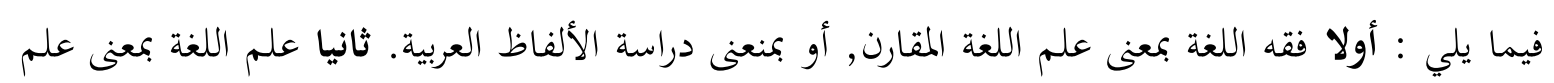

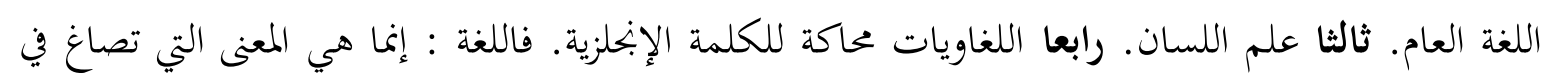

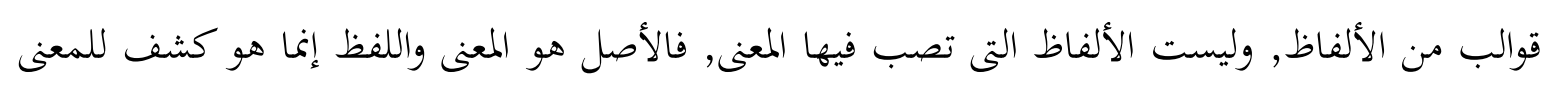

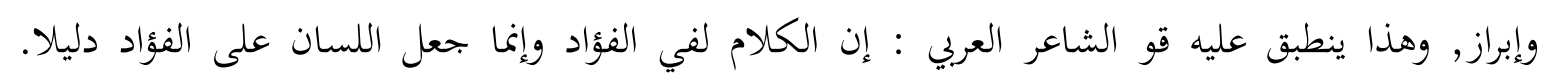

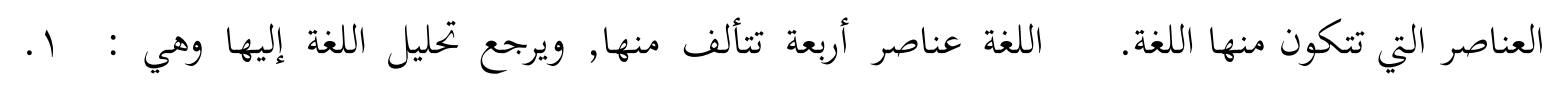

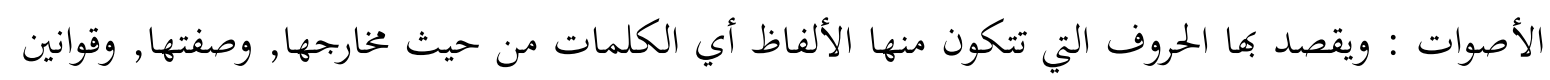

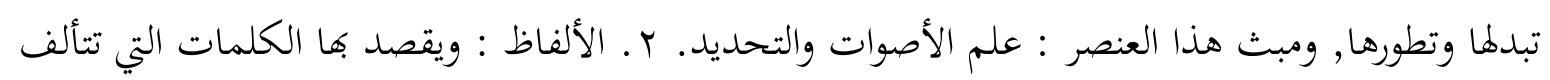

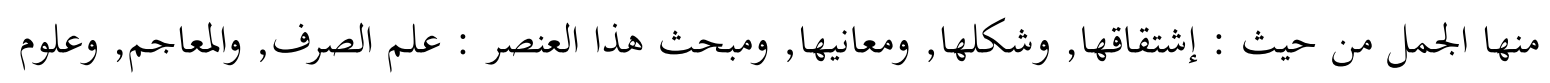

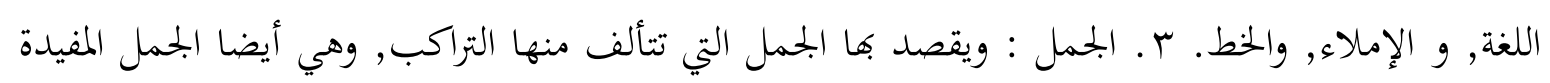

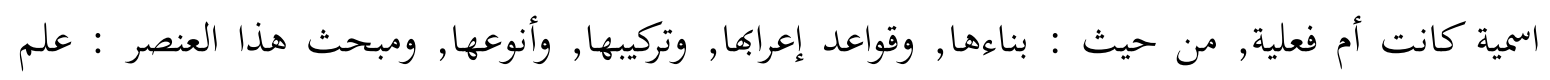

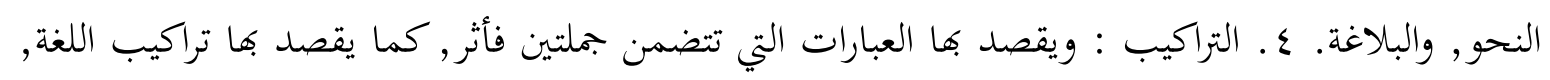

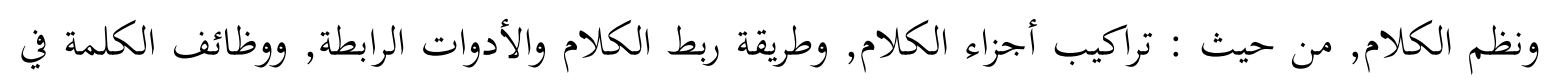

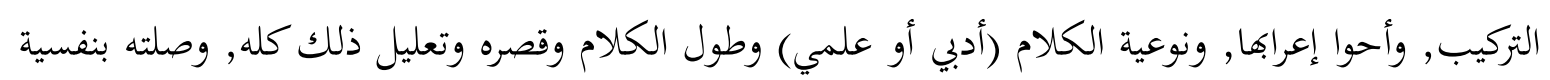

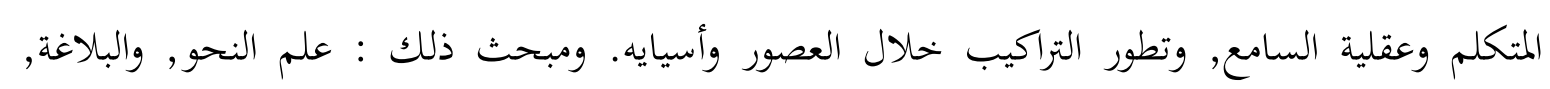

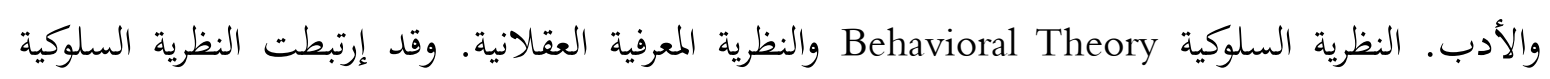

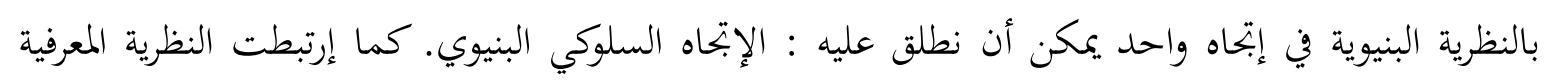

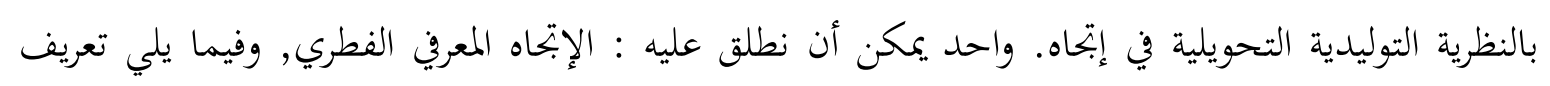


كذذين الإتجاهين وعلاقة كل منهما اللغات الأجنبية. الإتحاه السلوكي البنيوي, الإتحاه السلوكي البنوي واكتساب اللغات الأجنبية, الإتحاه العرفي في الفطري.

\section{الكلمات الئيسية: الثوجهات، فهم علم اللغة}

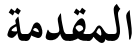

إن تعلم علم اللغة ليس بالأمر السهل و الهين. لكنه في البحث والدراسة أن يهتما التوجيهات التي

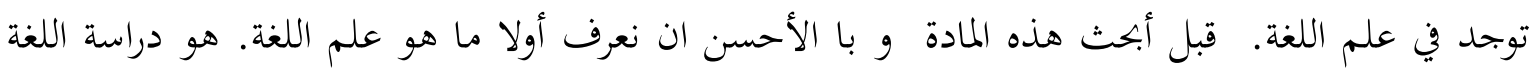

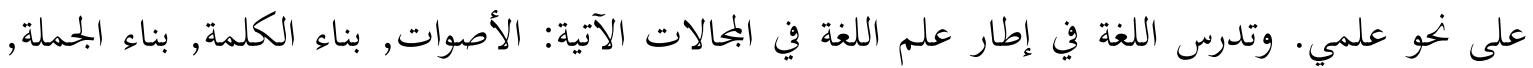
المفردات ودلالتها. وبعد أن نفهم تعربف علم اللغة جيدا. واضح لنا عن العناصر الموجودة في علم اللغة.

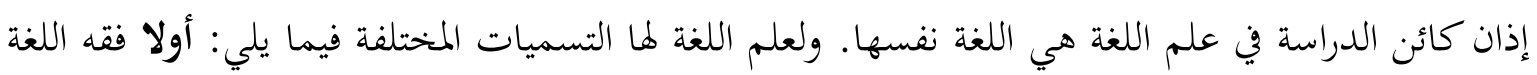

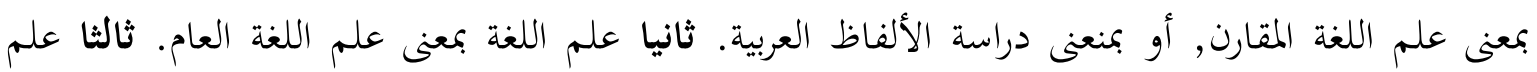

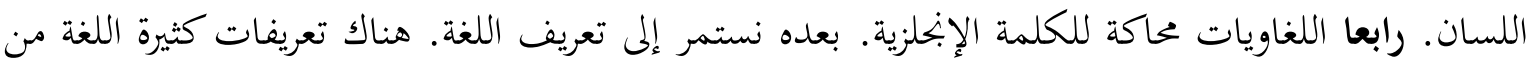

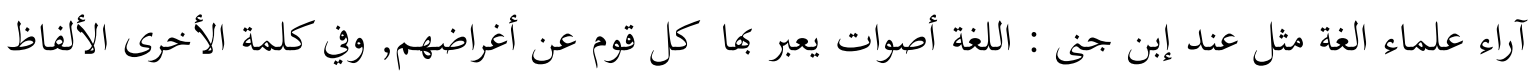

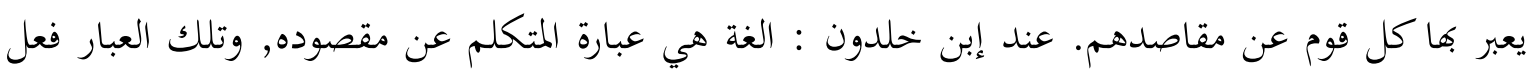

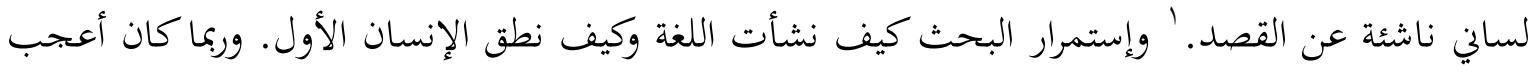

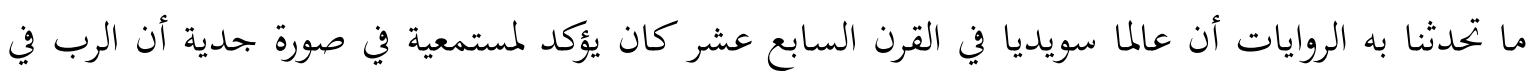

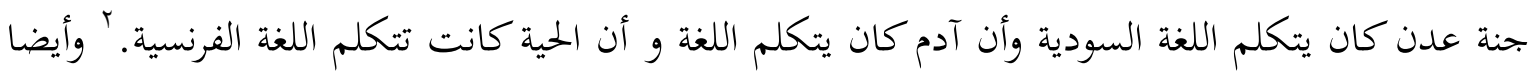

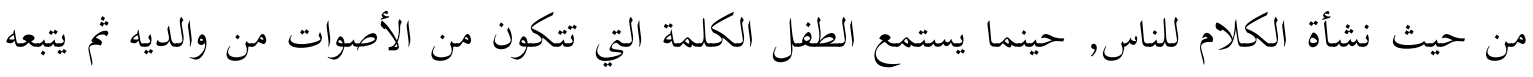

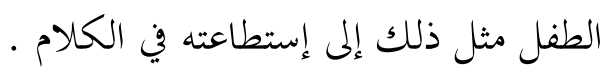

فروع علم اللغة

أشرنا في بداية الفصل إلى أن علم اللغة ينقسم قسمين أو فرعين أساسين هما : علم اللغة العام, أو

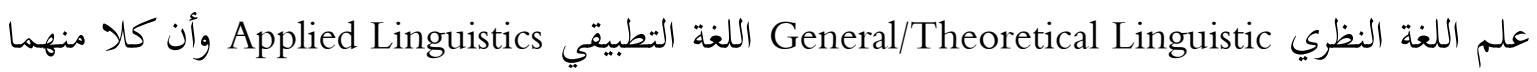
يندرج تتهه عدد من الفروع التي تتفرع أيضا إلى فروع صغيرة, وفيما يلي تعريف بهذين الفرعين الأساسين,

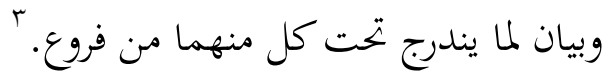

\footnotetext{
' د. محمود فهمى حجازى, علم اللغة العربية مدخل تارخي مقارن, القاهرة و ص. 9

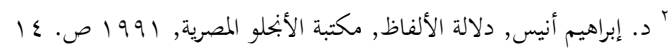

'العصيلي, النظريات اللغوية قالنفسية وتعليم اللغة العربية.
} 


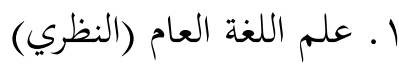

يعرف هذا العلم بأنه دراسة اللغة أي لغة دراسة نظرية وصفية تحليلية قائمة على أسس علمية ومناهج عامة, ويسعى إلى تطبيقها على دراسة ح اللغات جميعا, بصرف النظر عن فصائلها وتصنيفاتا العائلية, للخروج بنظرية لغوية عامة. وإذا أطلق مصطلح علم اللغة وحده, بحردا من أي وصف آخر فيقصد به عادة علم اللغة العام أو علم اللغة النظري. يشمل علم اللغة فرعا منها : علم اللغة التارخي, وعلم اللغة المقارن, وعلم اللغة الوصفي, وعلم اللغة الجغرين, وفقه اللغة, وعلم الأصوات (النطقي والسمعي و الفيزيائي), وعلم وظائف الأصوات, وعلم الصرف, وعلم النحو, وعلم الدلالة, وعلم المعاجم وعلم اللغة التداولي. r ا ب. علم اللغة التطبيقي ثمة جدل طويل حول تعريف هذا العلم, وحدوده, وبحالاته, وإستقلاله, وعلاقته بالعلوم الأخرى, والتفصيل في هذا الأمر يحتاج إلى بحث مستقل؛ . لكننا توخيا للاختصار يمكن أن نعرفه وa بأنه : فرع من فروع علم اللغة, يعني بالجانب التطبيقي منها, ويستفيد من علوم شيئ ذات صلة بدراسة اللغة واكتسابها وتعلمها وتعليمها, ومن تلك العلوم : علم التربية, وعلم النفس, وعلم الإجتماع, وعلم دراسة الأجناس البشرية وتنوع الثقافات Anthropology وعلم اللغة الإثنى (العرقي) Ethnolinguistics, وعلم المعلومات Education Media وعلم الحاسب الآلي Computer Science وتقنيات التعليم ووسائل الإتصال Comunication Means وغيرها. فعلم اللغة التطبيقي إذن يعنى بالجانب التطبيقي من اللغة, سواء كان التطبيق تعليما, وهو الغالب, أم كان غير تعليما, وهو الغالب, أم كان غير تعليم, وهو قليل, ويركز بوجه خاص على اكتساب اللغات الأجنبية وتعلمها وتعليمها. نشير هنا إلى نوع من التداخل بين بعض فروع علم اللغة العام وفروع علم اللغة التطبيقي, أي أن بعض هذه الفروع مشترك بين علم اللغة العام وعلم اللغة التطبيقي, بسبب تقارب في المصطلح أو في التطبيق. فعلم المعاجم (علم المفردات) مثلا, وهو علم نظري, قد يتداخل مع علم صناعة المعاجم الذي يعد علما تطبيقيا. وسواء ارتضينا الأول التصنيف الثاني في النظر إلى علم اللغة وفروعه, فإن هذا لا يغير من الأمر شيئا فيما يتعلق بتعليم اللغات. فميدان تعليم اللغة للناطقين بلغات أخرى في عصرنا الحاضر قد إستفاد من علم اللغة الحديث بجميع فروعه وميدانه النظري منها والتطبيقي. كما إستفاد من علوم أخرى, كعلم دراسة الأجناس البشرية وتنوع الثقفات, وعلم الإجتماع, وعلم النفس, ووعلم التربية, والثقافة الإسلامية, ومناهج البحث, وعلم الحاسوب, وعلم المعلومات, والإحصاء, والرياضيات. 
ظهر في العصر الحديث عدد من النظريات والإبحاهات التي تفاوتت في نظرتا إلى طبيعة اللغة, وأساليب تحليلها ودراستها, وطرائق إكتساها وتعلمها وتعليمها. من أبرز هذه النظريات والإبحاهات نظريتان في علم اللغة هما : النظرية البنوية Structural Theory والنظرية التوليدية التحولية Generative Theory Transformational العقلانية. وقد إرتبطت النظرية السلوكية بالنظرية البنيوية في إبجاه واحد يمكن أن نطلق عليه : الإبحاه السلوكي البنيوي. كما إرتبطت النظرية المعرفية بالنظرية التوليدية التحويلية في إبحاه. واحد يمكن أن نطلق عليه : الإبحاه المعريف الفطري, وفيما يلي تعريف بهذين الإتحاهين وعلاقة كل منهما اللغات الأجنبية.

\section{| الإتجاه السلوكي البنيوي}

أ. النظرية السلوكية

النظرية السلوكية هي إحدى نظريات التعليم التي ظهرت في ذهاية القران التاسع عشر وبداية القرن

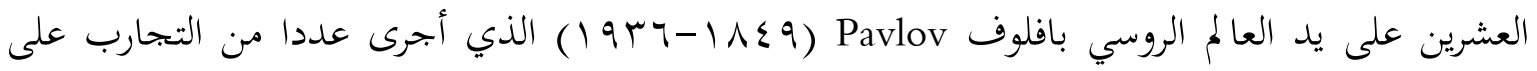
الحيونات بهدف تحديد إستجاباةما لمثيرات معينة, طمعا في الوصول إلى نظرية نعامة في التعلم لدى الحيوان وسلوكه من خلال التحكم في المثيرات الشرطية, وإستنتج منها ما يعرف يقانون المثير و الإستجابة. ثم تبع بافلوف في هذا الإتحاه بحموعة من علماء النفس الأمركيين منهم جون ب واطسون John B. Waston

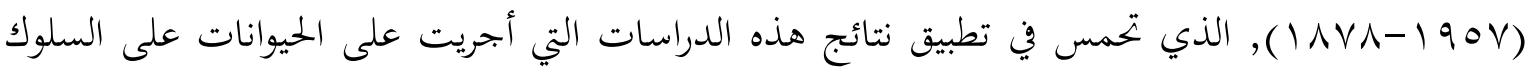
الإنساني .

إستخلص هؤلاء السلوكيون وغيرهم من أنصار هذا الإتجاه قوانين متعددة, طبقت في بحالات التعلم الإنساني في المدرسة والبحتمع. من أهم هذه القوانين قانون التكرار وقوانين أثر التعلم والتعليم, وقوانين الحوافز والثواب والعقاب وقانون إنتقال الأثر الشرطي, وقانون انطفاء الأثر الشرطي. ثم ظهرت آثار هذه القوانين على المناهج المدرسية من حيث المحتوى والطريقة, وانعكست على تقسيم التحصيل الدراسي والجوانب الشخصية لدى الطلاب. ' ثم إتجهت مجموعة من علماء النفس السلوكيين في الولايات المتحدة الأمركية إلى تطبيق هذه القوانين على إكتساب اللغة وتعلمها وتعليمها لإعتقادهم أن إكتساب اللغة لا يختلف عن إكتساب أي سلوك آخر . وإكتساب الطفل لغته الأم في نظر هؤلاء يقول على تكوين عادات سلوكية, ويعتمد على التعزيز الإجابي الذي يلتقاه من والديه أو من معلميه أو من غيرهم من المحيطين به, كلما نطق نطقا سليما, كما 
يعتمد في إستبعاد العادات السيئة على التعزيز السيلي الذي يتلقاه من هؤلاء عن طريق العقاب المباشر أو غير المباشر. فاللغة إذن عبارة عن مهارة تنمو عن طريق التعزيز الإجابي للإستجابات الصحيحة الذي يؤدي إلى لى

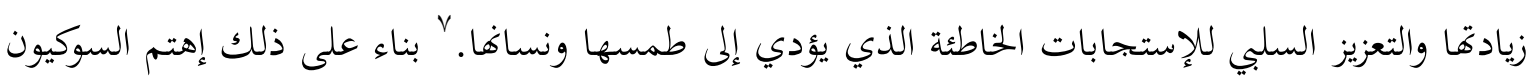
بالبيئة اللغوية المحيطة بالطفل بما في ذلك الولدان والمعلم والكتاب, معتقدين أن عقل الطفل صفحة بيضاء تسطر عليها البيئة ماتشاء, وأن هذه البيئة هي المصدر الوحيد الذي يعتمد عليه الطفل في إكتساب لغته الأم, مغفلين ما لديه من قدرات عقلية معرفية, وملكة لغوية خاصة به دون غيره من المخلوقات. وهذا لا يعنى بالضرورة أن السلوكيين لا يؤمنون بوجود العمليات العقلية ذات العلاقة باكتساب اللغة أو تعلمها, وإنما يعني

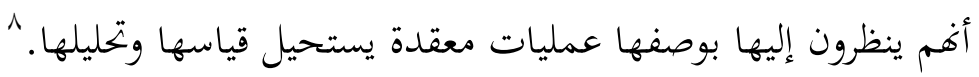

\section{ب. النظرية البنيوية الوصفية}

أسس هذا الإبحاه ووضع قواعده اللغوي السويسري فردينان دي سوسير ونسب إليه على الرغم من

وجود هذه الفاهيم والآراء لدى بعض معاصريه. لقد قامت على أساس من هذا الإبحاه الشكلي في التحليل اللغوي مناهج اللغوية أخرى. من هذه المناهج : التوزيعية التي هتم بدراسة سلوك العنصر اللغوية (الفونيمات والمورفيمات والمقاطع و و الكلمات والعبارات والجمل) من خلال المواقع التى تحتلها في الكلام. ومنها منهج التحليل إلى المكونات المباشرة الذي يرى أصحابه أن الجملة نسق منظوم على نخو مخصوص, وأن فهم التراكيب اللغوية يقوم على فهم هذا النسق المنظوم. وتتم عملية تحليل الجملة في هذا المنهج من خلال تقسيم الجملة إلى أقسام أساسية, ثم تقسيم كل قسم إلى أقسام فرعية. فجملة : الولد الصغير حفظ القران مثلا : يمكن تقسيمها إلى مبتدأ (الولد الصغير) خبر (حفظ القران), وتقسيم المبتدأ إلى إسم (الولد) وصفة (الصغير) وتقسيم الإسم إلى أداة تعريف (أل) وصفة نكرة (ولد) وهكذا حتى تنتهي الجملة. ومنها أيضا منهج الخانية

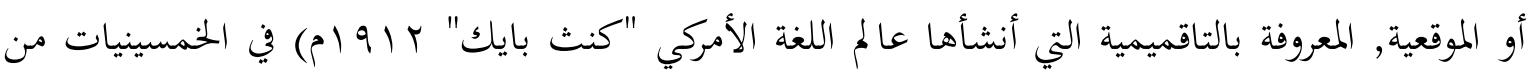
القرن العشرين بهدف تدريب اللغويين على التحليل اللغوي لأغراض الترجمة.

يقوم هذا المنهج على أساس تقسيم اللغة إلى ثلاثة أنظمة أساسيا هي : الأصوات, المفردات, والقواعد. لكل نظام من هذه الأنظمة بحموعة من المستويات وفي كل مستوى وحدات صغرى. فنظام القواعد مثلا يندرج نحته موستويات هي : المورفيم, والكلمة, والعبارة, والجملة, والفقرة, وتحت كل مستوى وحدات نهوية صغرى ذات معنى تسمى Tagmemes والجملة في هذا المنهج قال يتكون من بحموعة من الحقول (الخانات) أو المواقع, كالفاعل. والمفعول, والمبتدأ والخبر ونحو ذلك. ولكل حقل أو موقع مالئة, وهي عبارة 
عن نوع خاص من العناصر اللغوية التي يمكن أن تحتل تلك الحقل أو تقع في ذلك المقع. فالفاعل حقل يمكن أن تملاء با لإسم الظاهر أو بالضمير أو بالعبارة الإسمية أو بالمصدر المؤل والخبر حقل يمكن أن تملاء بالإسم الظاهر أو بالصفة أو بالعبارة الفعلية وهكذا في بقية والظائف النحوية.

\section{ج. الإتجاه السلوكي البنوي واكتساب اللغات الأجنبية}

رأينا في الفقرات السابقة كيف إقترنت النظرية ابنوية في علم اللغة, في فاية المطاف بنظرية نفسية معاصرة لما هي النظرية السلوكية واتحدت معها بشكل أطلقنا عليه : الإتحاه السلوكي البنوي. وقد تم ذلك عل بل ى يد أبرز علمائها وهو ليونارد بلومفيد الذي تأثر بآراء السلوكين فيالنظرة إلى طبيعة اللغة وأساليب إكتسابها,

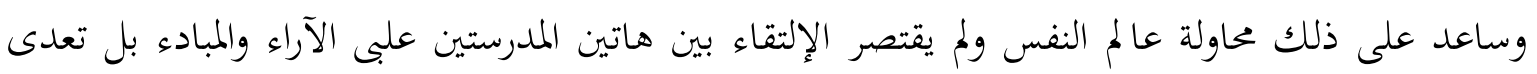
ذلك إلى بحالات لغوية تطبيقية في المتر والمدرسة وغيرهما من العوامل الخارجية التي تتحكم في نظر هؤلاء في تكوين اللغة الأم للطفل. تم إنتقلت هذه التطبيقات إلى ميدان تعليم اللغات لأجنبية والثانية, وبخاصة تعليم الإبحليزية بوصفها لغة ثانية في الولايات المتحدة الأمركية لأن القائمين على برامج تعليم اللغات الأجنبية كانوا من السلوكيين البنيوين.

وقد ركزت برامج تعليم اللغات الأجنبية في تلك الحقبة على لغة الحديث الشفهي, فقدمت مهارة

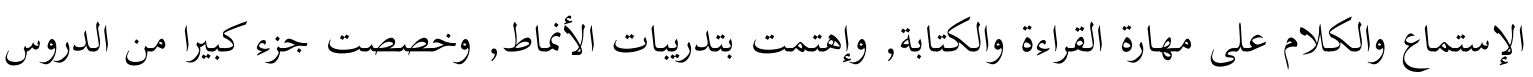
لهذا الجانب. وكان هدف من تدريبات الأنماط في نظر هؤلاء تعليم اللغة الهدف تكوين العادات اللغوية بطريقة لا شعورية من خلال تعليم القواعد من غير حاجة إلى شرح القواعد بطريقة نظرية مباشرة. نتيجة لتبنى اللغوين التطبيقيين المذهب السمعي الشفهي, نشأت طرائق وأساليب لتعليم اللغات الأجنبية, كان منها الطريقة المباشرة, والطريقة الطبعية, لكن أشهر هذه الطرائق وأوسعها إنتشارا الطريقة السمعية الشفوية, التي سيطرت على الميدان منذ أواخر الخسينيات حتى منتصف السبعييات من القرن العشرين. وتعد هذه الطريقة خير مايمثل هذا الإتحاه, ويصور الإلتقاء بين النظرية السلوكية في علم النفس والنظرية البنيوية في علم اللغة.

\section{ץ. الإتجاه العرفي في الفطري أ. النظرية المعرفية}

لم يكن الإهتمام بالنواحي المعرفية في بحال التعلم والتعليم وليد هذا العصر , بل كان هوالمنهج السائد قبل طهور النظريات النفسية المعاصرة. وقد شهد النصف الأول من القرن العشرين ظهور عدد من النظريات المعرفية التي كانت شائدة قبل ظهور النظريات السلوكية. من هذه النظريات : النظرية الكلية, الكلية, المعروفة بنظرية الجشتالت التي ترى أن إدراك المتعلم يتجه إلى الكل الذي هو نظام مترابط متسق, مكون من أجزاء 
متفاعلة, وسابق لأجزائه من الناحية المنطقية, كما ترى أن التعلم الحقيقي يعتمد على إدراك العلاقات بين

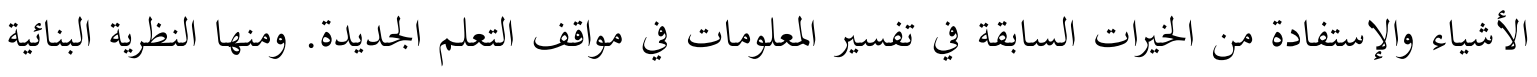

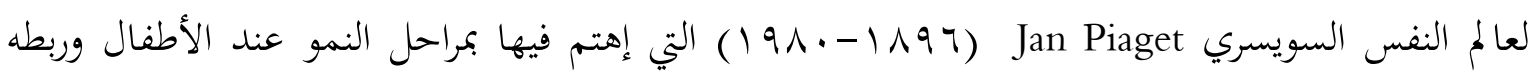
بالنمو العريف, ونبه إلى ضرورة التوازن في الإهتمام بين العوامل الداخلية والعوامل الخارجية في العملية العبلية التعليمية. وفيما يتعلق بإكتساب اللغة, يرى بياجيه

مفهوم اللغة

المعنى اللغوي للغة يعنى : الكلام الباطل الذي لا فائدة فيه, يقول رسول الله ص (من مس الحصا فقد

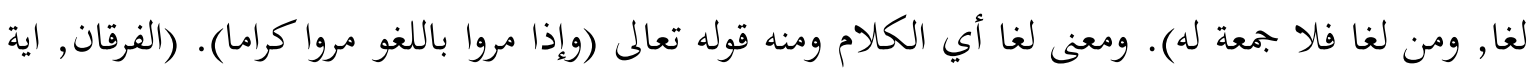
V :

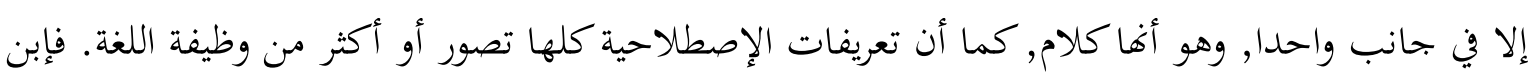

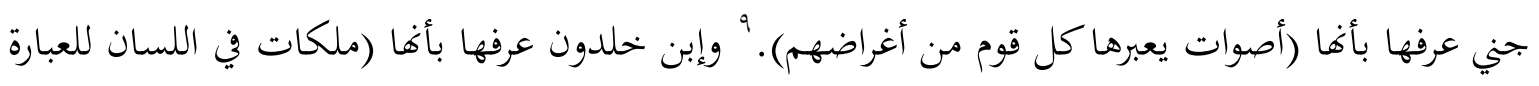

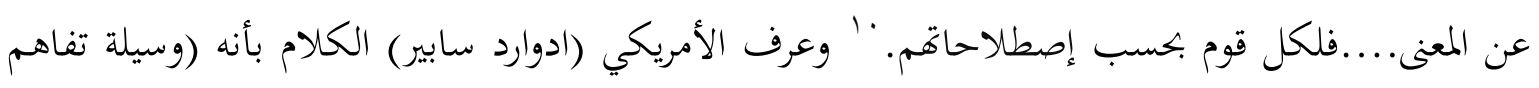

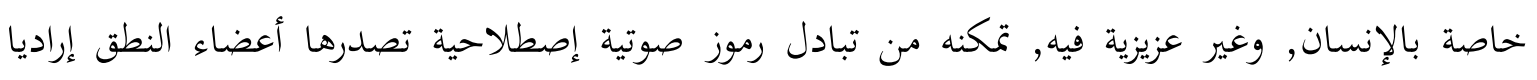

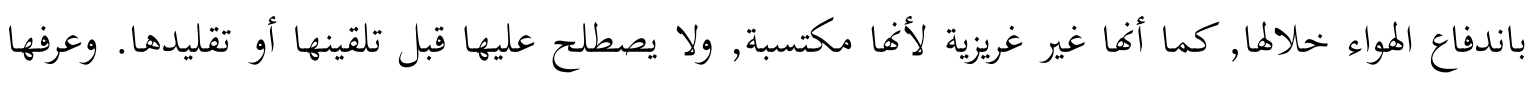

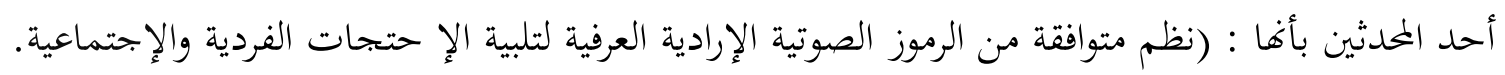

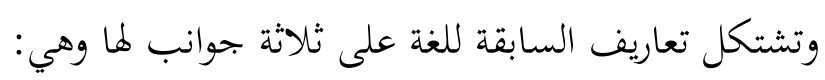
ا ـ الطبيعة الصوتية للغة

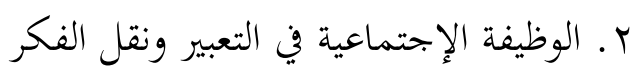

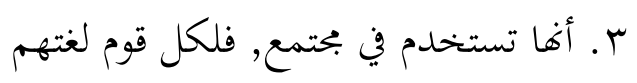

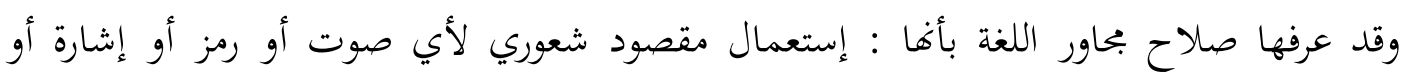

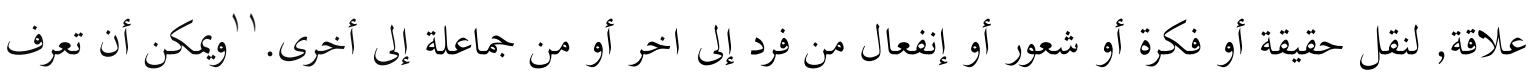

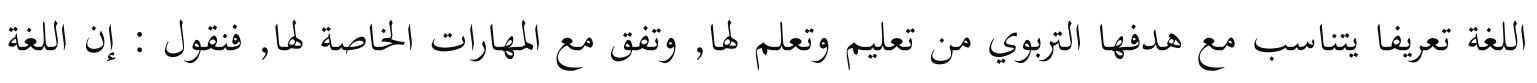

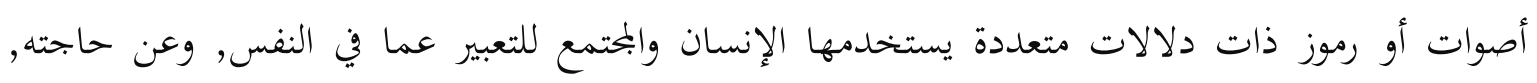

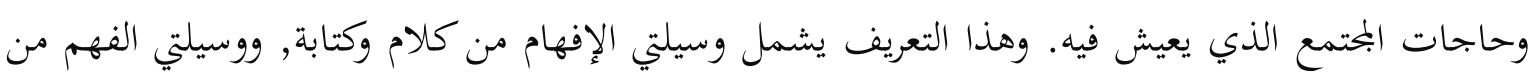

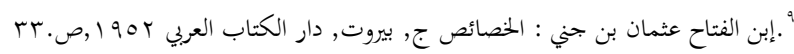

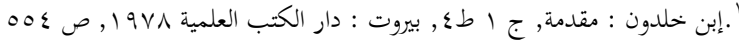

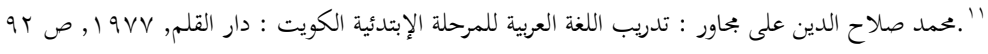


إستماع وقراءة, سواد أكان هذا عن طريق الرموز المنطوقة أم الرموز المكتوبة. ومن الإتجاهات الحديثة في فهم اللغة النظرة إليها على أها : المعنى لا الألفاظ. فاللغة : إنما هي المعنى التي تصاغ في قوالب من الألفاظ, وليست الألفاظ التى تصب فيها المعنى, فالأصل هو المعنى واللفظ إنما هو كشف للمعنى وإبراز, وهذا ينطبق عليه قو الشاعر العربي : إن الكالام لفي الفؤاد وإنما جعل اللسان على الفؤاد دليلا. العناصر التي تتكون منها اللغة اللغة عناصر أربعة تتألف منها, ويرجع تحليل اللغة إليها وهي : 1 ـ الأصوات : ويقصد بها الحروف التي تتكون منها الألفاظ أي الكلمات من حيث مخارجها, وصفتها, وقوانين تبدلها وتطورها, ومبث هذا العنصر : علم الأصوات والتحديد. r. الألفاظ : ويقصد بها الكلمات التي تتألف منها الجمل من حيث : إشتقاقها, وشكلها, ومعانيها, ومبحث هذا العنصر : علم الصرف, والمعاجم, وعلوم اللغة, و الإملاء, والخط.

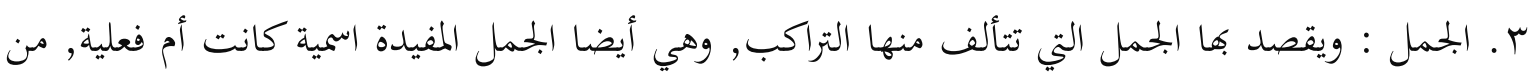
حيث : بناءها, وقواعد إعرابها, وتركيبها, وأنوعها, ومبحث هذا العنصر : علم النحو, والبلاغة ع. التراكيب : ويقصد بها العبارات التي تتضمن جملتين فأثر, كما يقصد بها تراكيب اللغة, ونظم الكلام, من ون حيث : تراكيب أجزاء الكلام, وطريقة ربط الكلام والأدوات الرابطة, ووظائف الكلمة في التركيب, وأحوا إعرابها, ونوعية الكلام (أدبي أو علمي) وطول الكلام وقصره وتعليل ذلك كله, وصلته بنفسية المتكلم وعقلية السامع, وتطور التراكيب خلال العصور وأسيايه. ومبحث ذلك : علم النحو, والبلاغة, والأدب.

\section{الخصائص العامة للغة}

الخصائص والسمات التي تتلاقي عليها لغات العالم يمكن حصرها وبلورةقا في العناصر التالية : ا ـ أغا بدأت صوتية ثم صارت رمزية مقروءة. فالإنسان في بداية حياته من لدن ادم إلى أن يرث الله الأرض

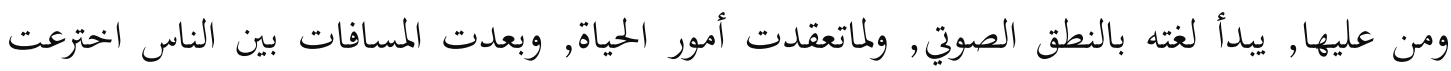
الكتاب, ولكل لغة رموزها الخاصة بها منطوقة أو مكتوبة. ورموز اللغة العربية هي حروفها : أ ب ت ث, ورموز اللغة الإنحلزية a b c وظاهرة التعبيرات الصوتية مشتركة بين الإنسان والحيوان والطير, ولكن الكلام هو أرقى التعبيرات الصوتية وهي خاصة لاتوجد إلا عند الإنسان وحده. r. أن اللغة نظام صوتيا ورمزيا : فبالرغم من أن لكل لغة نظامها الخاص بها نطقا أو كتابة, إلا أن اللغات جميعا تشترك في أن لها نظاما. ولا توجد لغة ليس لها نظام, فالنظام الصوتي يتمثل في النطق الصحيح 
للأحرف, وإخراج كل حرف من مخرجه, والنظام الرمزي في كتابة كل حرف. في كل لغة بشكل معين-

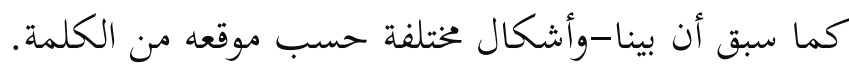

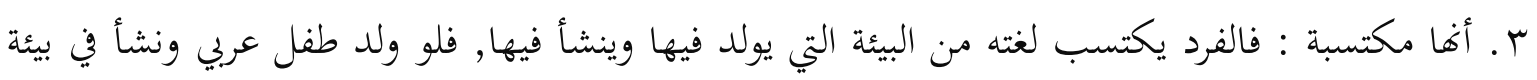
عربية لا كتسب اللغة العربية من ابيئة نشأ فيها.

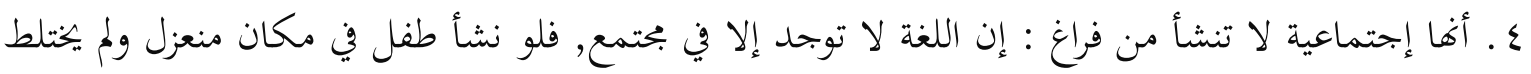

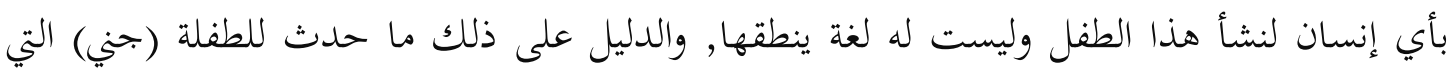

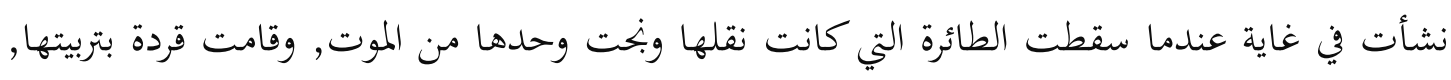

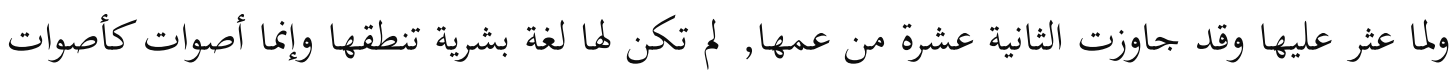

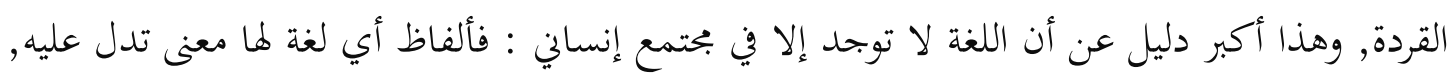

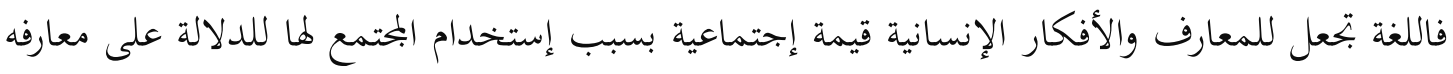
وأفكاره.

ه. أها نامية وحية : بمعنى أها في تفاعل مستمر على مر الأزمان, فهي نامية وحية في نفسها : تدخلها

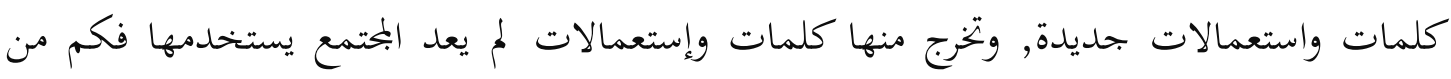

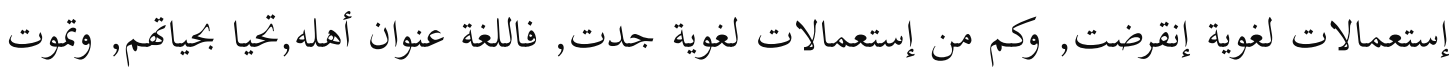
بموقم, وتتقدم وتتطور بتقدم وتطور هم, وتضعف وتتخلف بضعنفهم وتخلفهم.

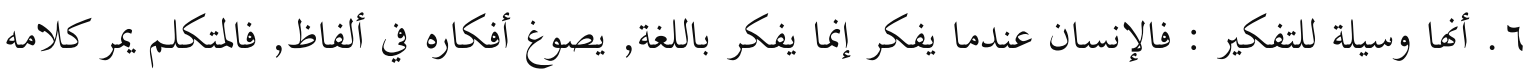

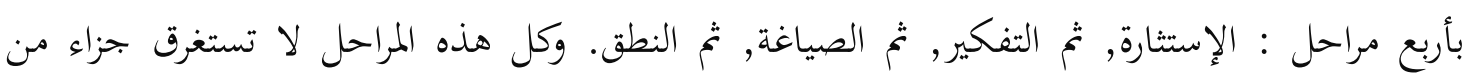

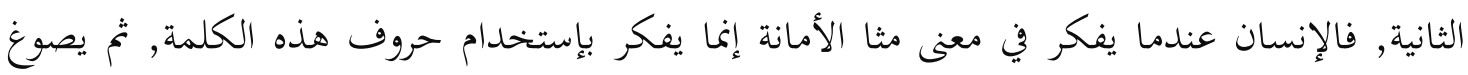

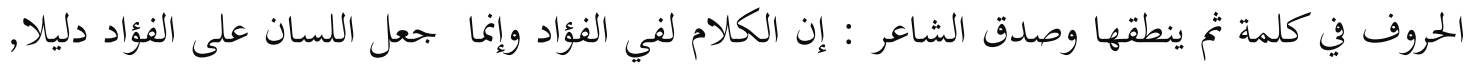

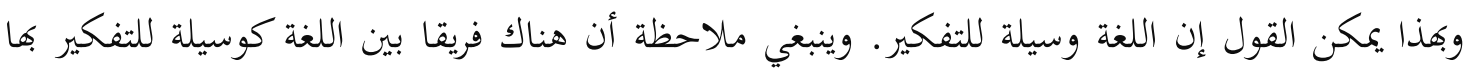

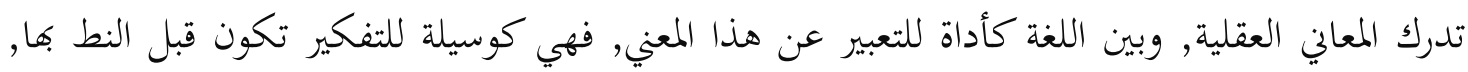

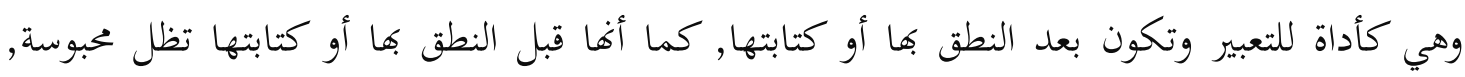

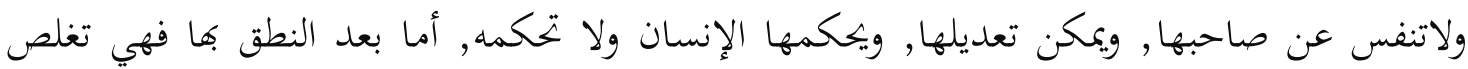

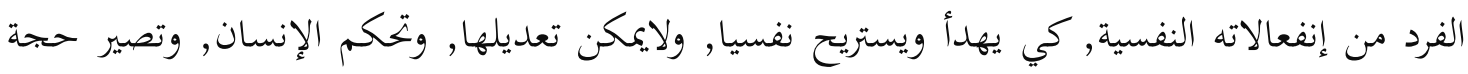

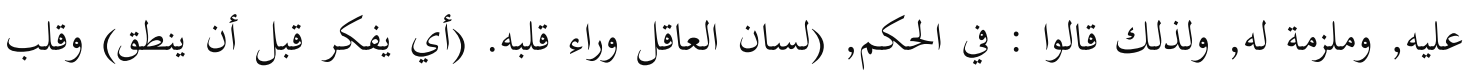

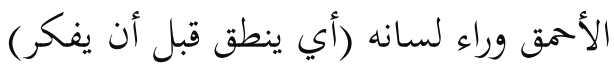


V. أخا تحمل معنى : فاللغة التي لا تحمل معنى لا تستحق أن تسمى لغة, بل هي بحرد أصوات جوفاء تسمى الهى

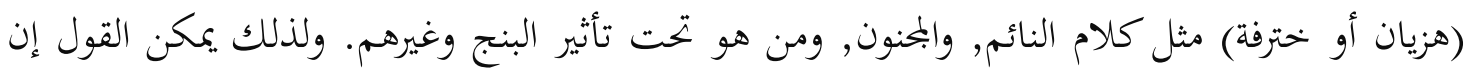

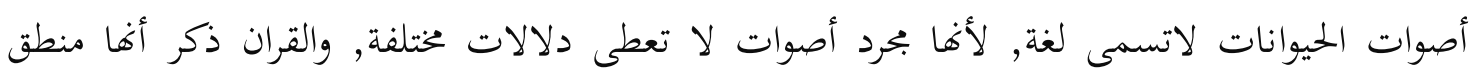
وليست لغة يقول الله سبحانه وتعالى (وورث سليمان داود وقال يأيها الناس علمنا منطق الطير ...) 1. أها إنسانية : أي أخها خاصة بالإنسان, فاللغة بحروفها المنطوقة والمكتوبة ولاتوجد إلا عند الإنسان فالله

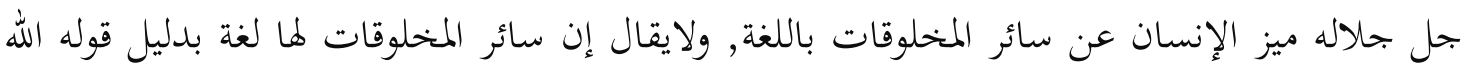

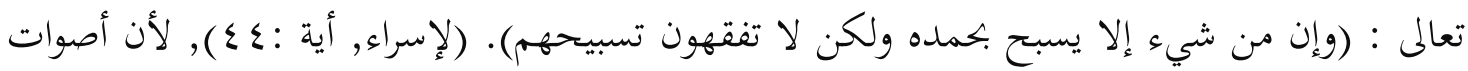

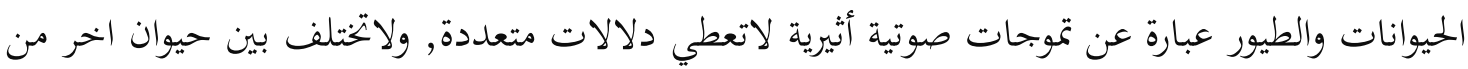
جنس واحد, ولا تختلف من مكان إلى مكان, ولكن الأصوات عند الإنسان تختبلف من إنسان لإنسان,

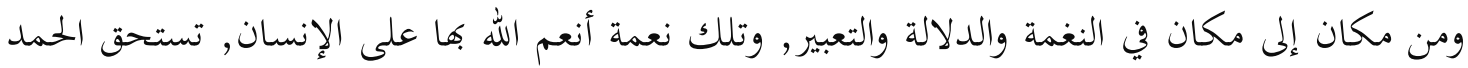
والشكر الله تعالى. أسرار اللغة

لم ينجز الإنسان عبر تاريخه الطويل شيئا مبدعا, وأكثر تأثيرا في حياته من إنشائه اللغة, وإستعمالها

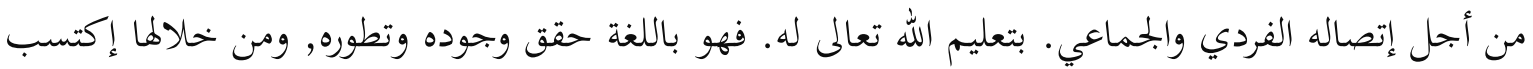

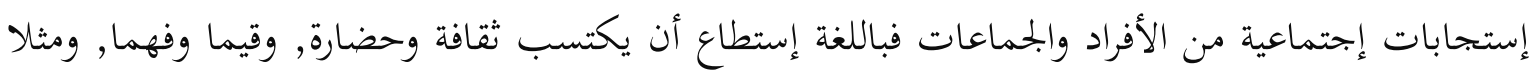

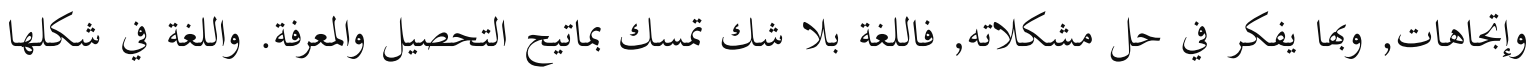

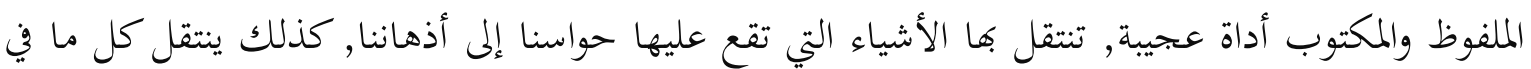

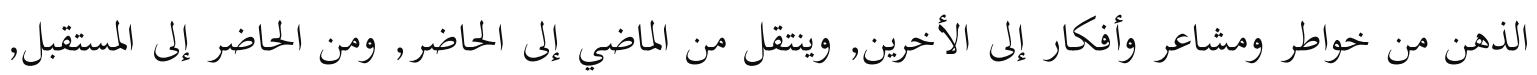

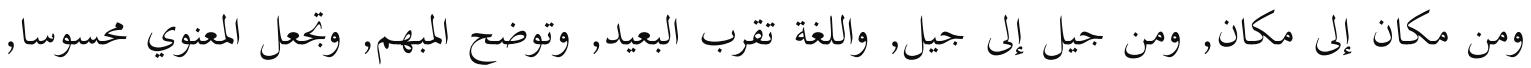
وابمهول معلوما. إلى غير ذلك من أسرار اللغة.

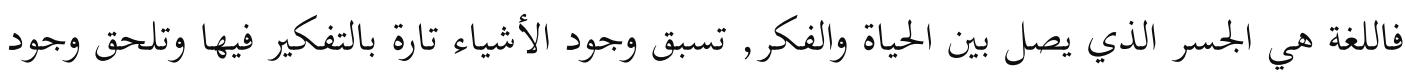

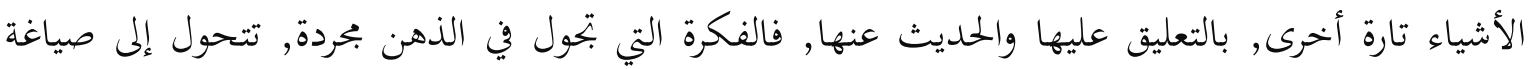

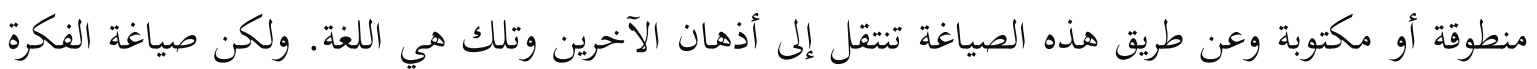

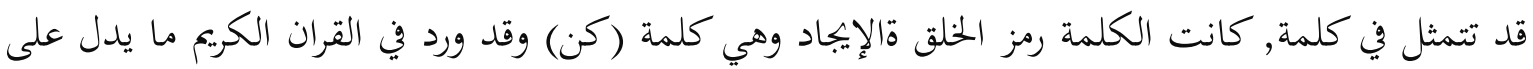

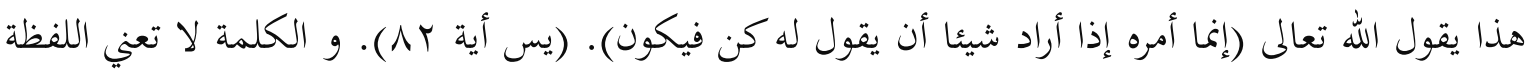

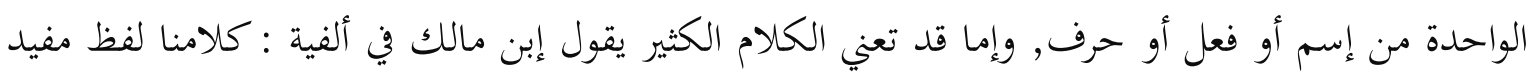

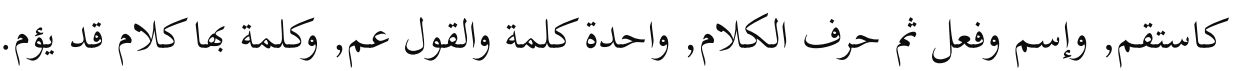


أي وكلمة قد يقصر بها كلام. وقد ورد في القران ما يدل على ذلك في الحديث عن ندم الإنسان العاصي يقول الله تعالى (حتى إذا جاء أحدهم الموت قال رب أرجعون, لعلي أعمل صالحا فيما تركت كلا إها كلمة هو قائلها...) (المؤمن أية : 99- . . 1). وورد في القران أيضا الكمة بمعنى العذاب يقول سبحانه (إن

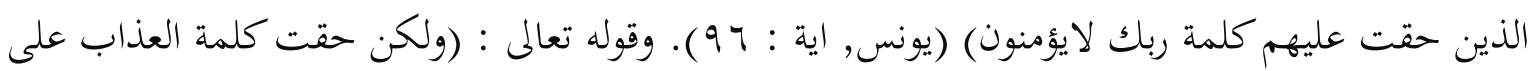
الكافرين) (الزمر , اية : VI كما تعنى الكلمة الخلق والإيجاد بقول الله تعالى (إنما المسيح عيسى بن مريم

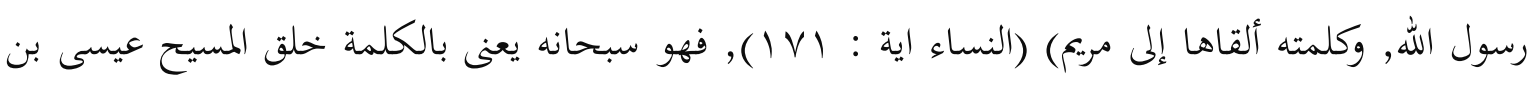

ولماكانت اللغة لهل هذه الأهمية في الحياة, كانت اللغة أداة السحر و البلاغة والفصاحة عند العرب, وجاء القران ببلاغته وفصاحته متحديا لهم, ويعد اللسان أداة أساسية للغة المطوقة وقد عرف العرب قديما أهيمة اللغة المنطوقة لاعتمادهم عليها, لذلك جعلوا اللسان نصف الحياة الإنسانية, يقول زهير بن أبي سلمي: لسان الفتى نصف ونصف فؤاده فلم يبق إلا صورة اللحم والدم. وكل هذا يكشف لنا بعض أسرار اللغة التي هي نصف الحياة الإنسانية, وتميز الإنسان عن غير من سائر المخلوقات.

\section{وظائف اللغة}

إن اللغة وظائف متعددة, منها ما هو متعلق بالفرد نفسه, ومنها ما هو متعلق بعلالاقة الفرد

$$
\begin{aligned}
& \text { بالمجتمع, ويمكن حصر هذه الوظائف فيما يلى : } \\
& \text { أ. وظيفة اللغة للفرد }
\end{aligned}
$$

ا . . وظيف شخصية : ويقصد بها قدرة وإمكانية الشخص (المتحدث أو الكاتب) على التعبير عن أفكاره الداخلية, وما يود إرازه من حقائق ومفاهيم, ومدركات لديه, بالأضافة إلى قدرته على التعبير عن عواطفه ومشاعره من : حب, أو بغض, أو سرور , أو حزن.....كما أن لكل شخص لغة خاصة به, في أسلوبه ونبرات صوته حتى أنه تعرف شخصية الإنسان من خلال صوته دون أن يرى عن طريق الهاتف مثلا, ولقد أثبت العلم الحديث أن لكل إنسان بصمة صوتية تختلف من إنسان لآخر , ولا يتفق فيها إنسان مع إنسان, مثل بصمة اليد, وصدق الله العظيم : (من ايته خلق السموات والأرض, واختلاف ألسنتكم

$$
\text { وألوانكم) (الروم, اية : ب r ب). }
$$

r ـ وظيفة نفسية : إن اللغة تؤثر في أدائها على نفسية الإنسان تأثيرا قد يكون مشجعا ومعززا, رافعا لمعنوياته, وقد يكون مثبطا, وخافضا للروح المعنوية, ذلك أن القدرة اللغوية وإستعمالها بنجاح بتعل الفرد سويا في نفسه, فالإنسان قد يشعر بالخحل, أو الخوف الذي يعجزه عن أداه اللغة أداء سليما, وقد إستخدمت

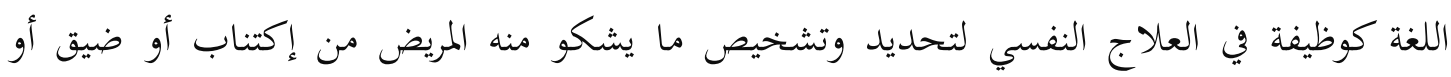


إضطراب أو عصبية, عن طريق كلامه عن نفسه. ولذا يجب تشجيع الأفرد المنعزلين الخجولين على

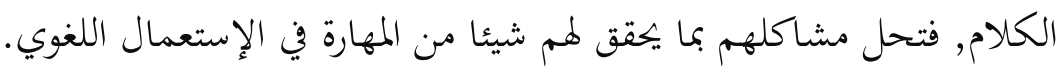

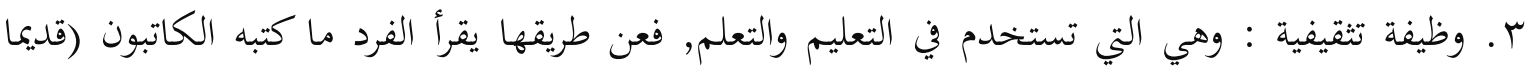

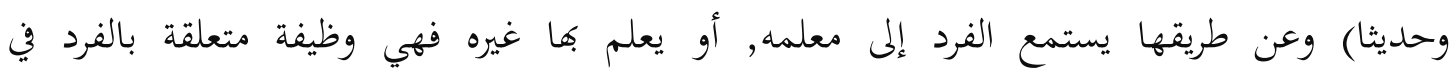

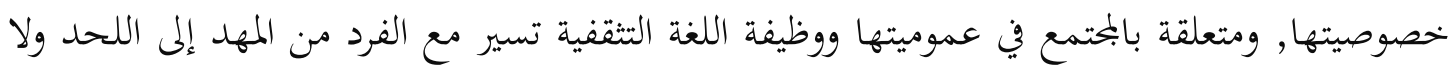

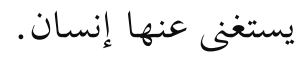

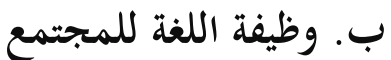
1. وظيفة إجتماعية تبادلية : ويقصد بها إستخدام اللغة في معاملات الناس اليومية : في المنزل, والشارع,

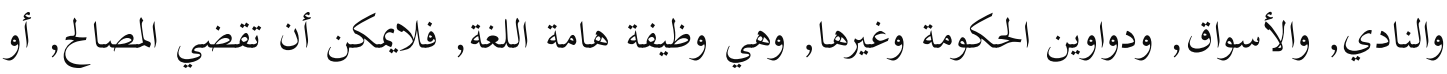

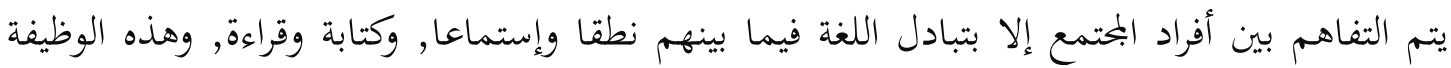

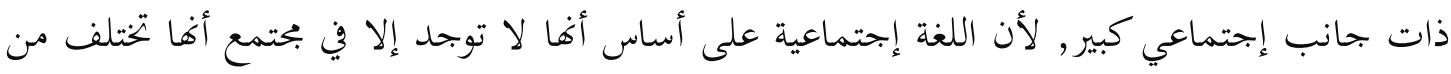

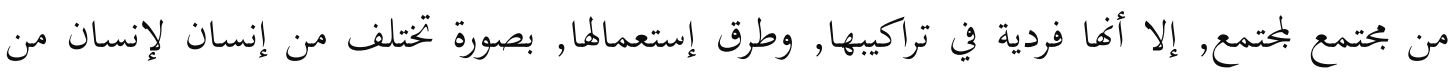

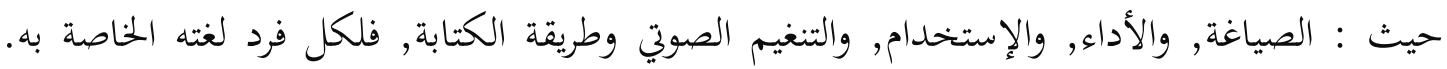
فاللغة إجتماعية من ناحية, وفردية من ناحية أخرى.

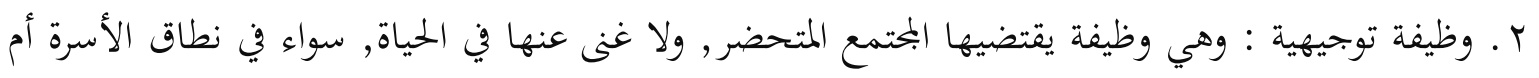

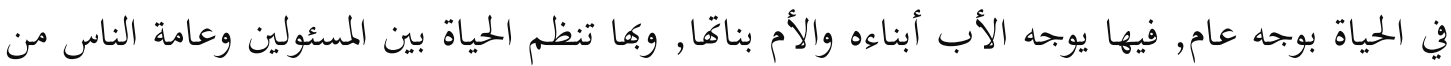
خلال الإرشادات المنطوقة والمكتوبة, هما يوجه رجال الأمر بالمعروف والنهي عن المنكر الناس إلى الخير المهاء

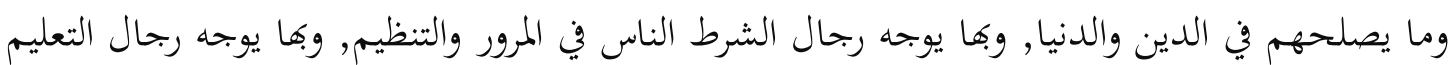

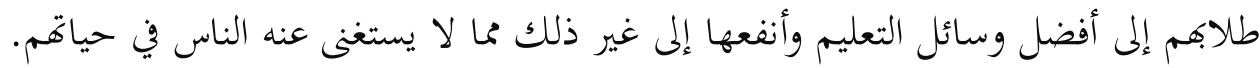

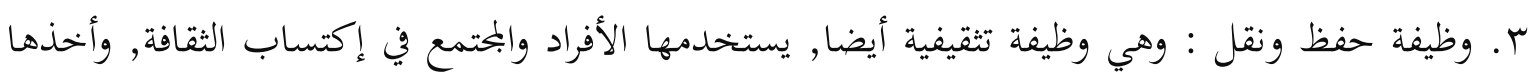

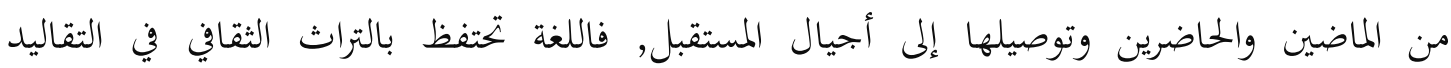
الإجتماعية, وتنقلها من جيل إلى جيل. 


\section{المراجع والمصادر}

د. محمود فهمى حجازى, علم اللغة العربية مدخل تارخي مقارن, القاهرة

د. إبراهيم أنيس, دلالة الألفاظ, مكتبة الأبحلو المصرية العصيلي, النظريات اللغوية النفسية وتعليم اللغة العربية

للدكتور عبده الراجحي بحث مفصل حول هذا الموضع في كتابه : علم اللغة التطبيقي وتعليم اللغة العربية, مطابع جامعة الإمام محمد الإمام محمد بن سعود الإسلامية لمزيد من الإطلاع على هذه الإتجاهات يمكن الرجوع إلى كتابنا : النظريات اللغوية والنفسية وتعلم اللغة العربية جورج غازدا, ويموند كورسيني, نظريات التعلم, دراسة مقارنة, عالم المعلرفة غازدا وكرسنى مرجع نفسه العصيلي, النظريات اللغوية, والنفسية وتعليم اللغة العربية

$$
\text { إبن الفتاح عثمان بن جني : الخصائص ج, بيروت, دار الكتاب العربي }
$$

حمد صلاح الدين على بحاور : تدريب اللغة العربية للمرحلة الإبتدئية الكويت : دار القلم

د أحمد فؤاد عليان, المهارات اللغوية, دار المسلم, رياض 